\title{
Controle biológico no manejo de Pratylenchus brachyurus em diferentes tratamentos na cultura da soja
}

\author{
Thayane Nascimento Coelho ${ }^{\mathfrak{a}}$, Warlyton Silva Martins ${ }^{\mathrm{b}^{*}}$, Flávia Fernandes Ribeiro de Miranda ${ }^{\mathrm{a}} \bullet$
}

a Centro Universitário Católica do Tocantins, Brasil

${ }^{\mathrm{b}}$ Universidade Estadual de Ponta Grossa, Brasil

*Autor correspondente (warlytonsilva@gmail.com)

\section{N F O}

\section{Keyworks}

biocontrol

root lesions

nematodes

Glycine max

\section{Palavras-chaves}

biocontrole

lesões radiculares

nematoides

Glycine max

\begin{abstract}
A B S T R A C T
Biological control in the management of Pratylenchus brachyurus under different treatments in soybean crop

The soybean culture has great importance for the Brazilian economy and may face biotic and abiotic barriers in its production, with nematodes being exponents in reducing the productivity of this culture. Thus, this work aimed to evaluate the effect of biological products for the control of Pratylenchus brachyurus in soybean culture. The experiment was carried out in the greenhouse and in the Phytopathology and Nematology Laboratory of University Center Católica do Tocantins. The experimental design was completely randomized, consisting of five treatments with different biological products: T1 - control (without treatment), T2 - Presence ${ }^{\circledR}$ (Bacillus subtilis and Bacillus licheniformis), T3 - Votivo Prime ${ }^{\circledR}$ (Bacillus firmus), T4 - Ecotrich ${ }^{\circledR}$ (Trichoderma harzianum) and T5 - Nemat ${ }^{\circledR}$ (Paecilomyces lilacinus) with four replications. To evaluate the efficiency of the biological control of nematodes, the soybean seeds were previously treated at the time of sowing. Seed treatment was carried out individually, adding the products according to the dosage established in the package insert. At 30 days after inoculation, characteristics, root length, plant size, number of nematodes and severity were evaluated. The products used did not affect the root length and the number of nematodes. The product T3

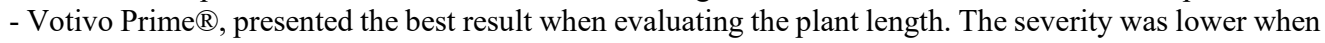
using some type of product for biological control.
\end{abstract}

\section{R E S U M O}

A cultura da soja possui grande importância para a economia brasileira e pode enfrentar entraves dos fatores bióticos e abióticos em sua produção, sendo os nematoides expoentes na redução da produtividade desta cultura. Desse modo, este trabalho teve como objetivo avaliar o efeito de produtos biológicos para o controle de Pratylenchus brachyurus na cultura da soja. O experimento foi realizado na casa de vegetação e no Laboratório de Fitopatologia e Nematologia do Centro Universitário Católica do Tocantins. O delineamento experimental foi inteiramente casualizado consistindo em cinco tratamentos com diferentes produtos biológicos: T1 - testemunha (sem tratamento), T2 - Presence ${ }^{\circledR}$ (Bacillus subtilis e Bacillus licheniformis), T3 - Votivo Prime ${ }^{\circledR}$ (Bacillus firmus), T4 - Ecotrich ${ }^{\circledR}$ (Trichoderma harzianum) e T5 Nemat ${ }^{\circledR}$ (Paecilomyces lilacinus) com quatro repetições. Para avaliação da eficiência do controle biológico de nematoides, procedeu-se o tratamento prévio das sementes de soja no momento da semeadura. $\mathrm{O}$ tratamento das sementes foi realizado individualmente, adicionando os produtos conforme dosagem estabelecida na recomendação da bula. Aos 30 dias após a inoculação, foram avaliadas a características, comprimento da raiz, tamanho de planta, número de nematoides e severidade. Os produtos utilizados não interferiram no comprimento da raiz e no número de nematoides. O produto T3 - Votivo Prime ${ }^{\text {, }}$, apresentou melhor resultado quando se avalia o comprimento de planta. A severidade foi menor quando se faz uso de algum tipo de produto para controle biológico. 


\section{INTRODUÇÃO}

A soja (Glycine max L.) é o grão mais importante produzido sob cultivo extensivo no Brasil (Melo e Brum, 2020). Atualmente, o Brasil é o maior produtor mundial de soja, segundo estimativas da safra 2019/2020 (Usda, 2020). Na safra de 2019/2020 a produção estimada foi de 121,09 milhões de toneladas (Conab, 2020).

Associada aos avanços em extensão de área e produtividade, a soja, assim como outras culturas, tem enfrentado diversos problemas fitossanitários. Os nematoides têm se apresentado como fatorchave no sucesso da lavoura, em detrimento de que estes podem ocasionar perda de produção, e, consequentemente, a qualidade final do produto (Costa et al., 2020).

Mais de 100 espécies de nematoides estão associadas ao cultivo de soja no mundo, fazendo com que $10,6 \%$ da produção mundial sejam perdidas em função do ataque desses parasitas de plantas (Soares e Dias, 2020). Dentre as espécies de importância para a cultura da soja no Brasil, destaca-se o Pratylenchus brachyurus, considerado, nos últimos anos, uma das espécies mais daninhas do gênero e de ocorrência comum no país (Cruz et al., 2020).

O nematoide da lesões radiculares (Pratylenchus brachyurus) tem causado danos econômicos à cultura no Brasil, principalmente na região CentroOeste, com perdas de produtividade de até $50 \%$. É conhecido como um nematoide endoparasita, possuindo também as características de migrador e polífago, agindo em razão da alimentação, movimentação e injeção de toxinas no interior dos tecidos, provocando assim danos as raízes (Silva et al., 2018).

Para prevenir ou realizar um controle das doenças que afetam a soja, diferentes estratégias podem ser utilizadas, dentre elas, o controle biológico, que é definido pelo uso de um ou mais organismos, ou parte deles, para reduzir ou controlar a população de pragas que causam doenças geradoras de efeito negativo na cultura (Machado e Costa, 2018).

O controle biológico se tornou uma nova alternativa que possibilita interação de diversos inimigos naturais dos nematoides fitófagos, com fungos, como o Trichoderma sp. que é considerado fungo antagonista e devido sua capacidade de degradar quitina realiza o controle dos nematoides atuando sobre os ovos e, bactérias, como o Bacillus, na formação de endósporos, antagonismo, produção de enzimas líticas, produção de siderófaros, solubilização de fósforo e fixação de nutrientes, tornam essas bactérias excelentes candidatas para a formulação de bioprodutos. $\mathrm{O}$ funcionamento dos agentes biológicos tem como característica a limitação ou estabilização dos nematoides, esta ocorre por meio da competição, bem como parasitismo e produção de compostos tóxicos (Santos et al., 2019).

Diante ao exposto, objetivou-se na presente pesquisa avaliar o efeito de diferentes produtos biológicos no controle de Pratylenchus brachyurus na cultura da soja na região do cerrado.

\section{MATERIAL E MÉTODOS}

\section{Local de pesquisa}

O experimento foi conduzido em laboratório de fitopatologia e casa de vegetação do Centro Universitário Católica do Tocantins - UniCatólica, Campus de Ciências Agrárias e Ambientais em Palmas - TO, no período compreendido entre abril e junho de 2019.

\section{Delineamento experimental}

Adotou-se o delineamento experimental inteiramente aleatorizado, consistindo em cinco tratamentos (produtos biológicos): T1 - testemunha (sem tratamento); T2 - Presence ${ }^{\circledR}$ (Bacillus subtilis linhagem QST 713, 1,0 x $10^{11} \mathrm{UFC} / \mathrm{g}$ e Bacillus licheniformis linhagem FMCH001, 1,0 x 1011 UFC/g); T3 - Votivo Prime ${ }^{\circledR}$ (Bacillus firmus cepa $\left.\mathrm{I}-1582,4,8 \times 10^{9} \mathrm{UFC} / \mathrm{g}\right) ; \mathrm{T} 4$ - Ecotrich ${ }^{\circledR}$ (Trichoderma harzianum Isolado IBLF 006, $1 \times 10^{10}$ UFC/g) e T5 - Nemat ${ }^{\circledR}$ (Paecilomyces lilacinus Isolado UEL Pae, $107,5 \times 10^{9} \mathrm{UFC} / \mathrm{g}$ ) com quatro repetições.

\section{Obtenção do inóculo}

O inóculo de Pratylenchus brachyurus foi obtido por meio da APROSMAT- Associação dos Produtores de sementes de Mato Grosso, localizada em Rondonópolis - MT.

\section{Preparo do solo, tratamento de sementes e plantio}

A cultivar de soja utilizada no presente estudo foi a cultivar 96Y90® da empresa Pionner.

Realizou-se o tratamento das sementes individualmente, adicionando os produtos conforme dosagens estabelecidas e, posteriormente, homogeneizadas para a melhor distribuição. A dosagem foi baseada na recomendação de cada produto, respectivamente: Presence ${ }^{\circledR}$ (150 gramas do produto para $100 \mathrm{~kg}$ de sementes), Votivo Prime ${ }^{\circledR}(200 \mathrm{~mL}$ do produto para $100 \mathrm{~kg}$ de sementes), Ecotrich ${ }^{\circledR}(50$ gramas de produto para $100 \mathrm{~kg}$ de sementes) e o Nemat ${ }^{\circledR}(100$ gramas do produto para $100 \mathrm{~kg}$ de sementes).

A semeadura de cinco sementes foi realizada em vasos com capacidade para 1 litro, em uma mistura contendo terra preta, areia lavada e substrato 
Bioplant ${ }^{\circledR}$, na proporção de 1:1:1, autoclavados a $120^{\circ} \mathrm{C}$ e $1 \mathrm{~atm}$, por 1 hora.

\section{Método de extração}

As extrações foram realizadas com a metodologia segundo Jenkins (1964), também conhecido como "método do peneiramento combinado à flutuação em centrífuga com solução em sacarose".

As amostras de solo e raiz foram homogeneizadas, em seguida adicionada água equivalente a 8 vezes a quantidade da amostra, vertendo a suspensão em peneiras número 20 (malha de 0,84 mm) para que se retirasse os resíduos maiores, logo em seguida, realizou o mesmo processo de peneiramento utilizando uma peneira número 400 (malha de $0,037 \mathrm{~mm}$ ).

Com o auxílio de uma pisseta, lavou-se a peneira recuperando todo o material nela contida, transferindo-os para um béquer. Após, dividiu-se o conteúdo do béquer em tubos de ensaio, e estes alocados em centrífuga por 5 minutos a aproximadamente $2.000 \mathrm{rpm}$.

Posteriormente, retirou-se o sobrenadante com o auxílio de papel absorvente, adicionando solução de sacarose (400 g de açúcar em $750 \mathrm{ml}$ de água), retornando à centrifuga por mais 1 minuto com a mesma rotação. Em seguida, verteu-se o líquido dos tubos sobre a peneira número 400 , fazendo a recuperação do material com auxílio de uma pisseta com água.

Obtida a suspensão final, uma alíquota de $2 \mathrm{ml}$ foi colocada em lâmina de contagem (Peters) e levada à microscopia óptica compatível (4 a 10x) para a quantificação dos nematoides.

Inoculação dos nematoides

A inoculação foi realizada no ato da semeadura, sendo inoculados $5 \mathrm{~mL}$ da suspensão, contendo 3 mil ovos e J2, próximo de cada semente. Transcorridos 30 dias após a inoculação, avaliou-se as seguintes variáveis:

Comprimento de raiz (CR): obtido através da mensuração desde o colo da planta até o final da raiz pivotante, realizado com auxílio de uma trena e os dados expressos em centímetros $(\mathrm{cm})$.

Altura de planta $(A P)$ : obtida com auxílio de uma trena, medindo do colo da planta até última folha verdadeira e os dados expressos em centímetros $(\mathrm{cm})$.

Número de nematoides: obtido através da extração do solo e raiz proposta por Jenkins (1964), em que a contagem foi realizada 4 vezes em cada amostra com uma alíquota de $2 \mathrm{ml}$ retirada e alocada em lâmina de contagem (Peters) que, posteriormente, foi levada à microscopia óptica compatível (4 a 10x) para a quantificação

Severidade: a severidade causada por Pratylenchus brachyurus foi dada através de escala de notas elaborada por Figueiredo (2013), com notas de 0 a 5 , conforme figura 1 .

\begin{tabular}{|c|c|c|c|c|c|}
\hline \multicolumn{6}{|c|}{ Escala de Notas } \\
\hline 0 & 1 & 2 & 3 & 4 & 5 \\
\hline 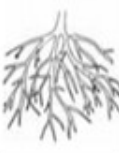 & 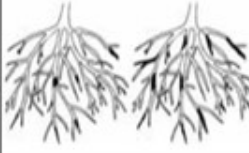 & 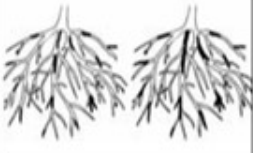 & 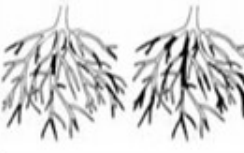 & 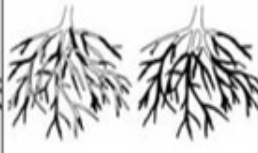 & 7 a \\
\hline $\begin{array}{c}0 \% \text { de } \\
\text { segmentos } \\
\text { de raizes } \\
\text { lesionadas }\end{array}$ & $\begin{array}{c}0,1-15,0 \% \text { de segmentos } \\
\text { de raizes lesionadas }\end{array}$ & $\begin{array}{c}15,1-25,0 \% \text { de segmentos } \\
\text { de raizes lesionadas }\end{array}$ & $\begin{array}{c}25,1-50,0 \% \text { de segmentos } \\
\text { de raizes lesionadas }\end{array}$ & $\begin{array}{c}50,1-85,0 \% \text { de segmentos } \\
\text { de raizes lesionadas }\end{array}$ & $\begin{array}{c}>85,0 \% \text { de } \\
\text { segmentos } \\
\text { de raizes } \\
\text { lesionadas }\end{array}$ \\
\hline
\end{tabular}

Figura 1 - Escala proposta para a avaliação, a campo, do grau de infecção de raízes de soja por Pratylenchus brachyurus, contendo seis categorias, de acordo com a percentagem da área das raízes lesionadas. Fonte: Figueiredo (2013).

\section{Análise Estatística}

Os dados obtidos foram submetidos à análise de variância utilizando o software AGROESTAT 1.0 (Barbosa e Maldonado Júnior, 2015) e as médias comparadas pelo teste de Scott- Knott ao nível de $5 \%$ de probabilidade. Os dados de severidade foram transformados em raiz de $\mathrm{x}+1(\sqrt{\mathrm{x}}+1)$.

\section{RESULTADOS E DISCUSSÃO}

A tabela 1 apresenta o resumo da análise de variância para comprimento de raiz, tamanho de planta, número de nematoides e severidade. Para o comprimento da raiz e número de nematoides os resultados não foram significativos. Já para o tamanho de planta e a severidade houve significância a nível de $1 \%$ de probabilidade $(\mathrm{p}<0,01)$. 
Tabela 1 - Resumo da análise de variância para comprimento de raiz (CR), tamanho de planta (TP), número de nematoides (NN) e severidade (SE) sob o uso de diferentes produtos biológicos.

\begin{tabular}{ccccc}
\hline \multirow{2}{*}{ CAUSAS DE VARIAÇÃO } & \multicolumn{4}{c}{ QUADRO DE ANÁLISE } \\
\cline { 2 - 5 } & CR & TP & NN & SE \\
\hline Produtos & $1,04 \mathrm{NS}$ & $8,92^{* *}$ & $0,67 \mathrm{NS}$ & $16,21^{* *}$ \\
\hline CV (\%) & $\mathbf{1 5 , 1 3}$ & $\mathbf{1 4 , 0 9}$ & $\mathbf{1 4 , 6 0}$ & $\mathbf{2 9 , 0 5}$ \\
\hline
\end{tabular}

** significativo ao nível de $1 \%$ de probabilidade $(\mathrm{p}<0,01),{ }^{*}$ significativo ao nível de $5 \%$ de probabilidade $(0,01=<\mathrm{p}<0,05)$ e ns não significativo $(\mathrm{p}>=0,05)$, respectivamente, pelo teste $\mathrm{F}$.

Na tabela 2 encontra-se os resultados numéricos para comprimento de raiz e número de nematoides (não houve diferenças significativas entre os produtos biológicos utilizados); e os resultados médios para o tamanho de planta e severidade. A não significância encontrada para estas variáveis no presente estudo corroboram com estudos realizados por Oliveira et al. (2011) em cana-de-açúcar, os quais objetivando o controle de nematoides do gênero Pratylenchus com os produtos carbofuran, fipronil, P. lilacinus, thiamethoxam e a interação destes, observaram que os tratamentos não diferiram entre si. Os autores concluíram tais resultados foram atribuídos ao fato de as plantas terem sido colhidas ainda jovens e devido o experimento ter sido conduzido em vasos.

Efeitos similares aos observados no presente trabalho com uma planta leguminosa foram obtidos por Araújo et al. (2012), estudando a eficácia de diferentes métodos e genótipos de soja no controle de Meloidogyne spp., onde os pesquisadores notaram que o tratamento químico utilizando carbofuran e tratamento biológico com Bacillus subtilis, ambos via TS, foram eficazes quanto a redução do desenvolvimento dos nematoides no genótipo suscetível.

Tabela 2 - Comparação estatística das médias para comprimento de raiz (CR), tamanho de planta (TP), número de nematoides $(\mathrm{NN})$ e severidade (SE) sob o uso de diferentes produtos biológicos.

\begin{tabular}{ccccc}
\hline \multirow{2}{*}{ Produtos } & $\mathbf{C R}$ & $\mathbf{T P}$ & $\mathbf{N N}$ & $\mathbf{S E}$ \\
\cline { 2 - 5 } & $\mathbf{c m}$ & $\mathbf{( c m )}$ & $\mathbf{( c m}^{\mathbf{3}} \mathbf{2}$ & Escala de notas \\
\hline Testemunha & 32,50 & $35,50 \mathrm{~b}$ & 40,00 & $3,25 \mathrm{a}$ \\
Presence ${ }^{\circledR}$ & 37,00 & $39,00 \mathrm{~b}$ & 42,00 & $1,25 \mathrm{~b}$ \\
Votivo Prime ${ }^{\circledR}$ & 34,75 & $56,75 \mathrm{a}$ & 40,25 & $0,87 \mathrm{~b}$ \\
Ecotrich & 40,00 & $40,50 \mathrm{~b}$ & 36,00 & $1,37 \mathrm{~b}$ \\
Nemat ${ }^{\circledR}$ & 36,75 & $36,00 \mathrm{~b}$ & 37,75 & $1,27 \mathrm{~b}$ \\
\hline
\end{tabular}

As médias seguidas pela mesma letra, na coluna, não diferem estatisticamente entre si. Foi aplicado o Teste de Tukey ao nível de $5 \%$ de probabilidade.

Observou-se, conforme a tabela 2, que amostras É possível observar que o produto Ecotrich ${ }^{\circledR}$ apresentou a maior média numérica para comprimento de raiz, apesar da não significância. Alguns estudos mostram que o fungo Trichoderma sp. (base do produto Ecotrich ${ }^{\circledR}$ ) é capaz de produzir hormônios, vitaminas e ácidos orgânicos que solubilizam o fosfato. Esse fungo pode fornecer micronutrientes e minerais à planta; produzem metabólitos secundários e colonizam a rizosfera da planta, interferindo positivamente em seu crescimento e ainda é capaz de induzir mudanças metabólicas que ativam o sistema de defesa da planta (Rezende, 2011).

Para a variável tamanho de planta apenas o produto Votivo Prime ${ }^{\circledR}$ se diferiu estatisticamente dos demais, com plantas apresentando em média 56,75 centímetros. Tais achados estão de acordo com os resultados de Mendonza et al. (2008), ao demonstrarem que o uso de células puras do Bacillus firmus para tratamento de plantas causam uma redução de galhas de Meloidogyne, além de estimular o crescimento das plantas.

Embora a não significância estatística, para o número de nematoides, observa-se numericamente que as plantas apresentaram médias semelhantes para todos os produtos utilizados. Essa ocasionalidade pode ser explicada por Grigolli e Asmus (2014). Os autores afirmam que até o momento não existem evidências de nematicidas que possuam potencial para reduzir significativamente a população de nematoides, entretanto estudos mostram que alguns produtos quando aplicados em tratamentos de sementes apresentam efeito supressor no início do desenvolvimento da cultura, em diversos casos, reduzem consideravelmente as perdas de produção.

Por outro lado, ao relacionar o número de 
nematoides com a característica severidade, percebe-se que a testemunha quando comparada aos demais se diferiu estatisticamente, apresentando maior severidade. Portanto, ainda que os produtos utilizados não reduzissem de maneira significativa a população de nematoides, foi possível minimizar o efeito severo exercido sobre a plantas com o emprego destes produtos.

Para Sundararaju e Jeyabaskaran (2003), o tipo de solo apresenta significativa influência na infecção e na severidade das necroses causadas nas raízes de bananeira por $P$. coffeae. O máximo de severidade de lesões em raízes e de reprodução do nematoide foi observado em solos silto-argilosos e aluviais, enquanto o minímo ocorreu em solos com baixa porosidade. Contudo, não é possível detectar nenhuma correlação entre os dois tipos de solo e a população do nematoide no solo ou nas raízes.

Observa-se que alguns nematicidas utilizados no controle biológico garantem a manutenção da produtividade, reduzindo os danos que a presença de nematoides provoca nas raízes das plantas (Koenning, 2007). Em estudos realizados por Schimidt et al. (2010), foi comprovado que o uso de produtos como o Bacillus subtilis no controle de nematoides em algodoeiros, foi bastante promissor, sendo possível a inclusão em estratégias de controle na cultura.

\section{CONCLUSÃO}

Os produtos analisados apresentaram resultados promissores quanto a redução do índice de severidade do nematoide Pratylenchus brachyurus na cultura da soja.

\section{REFERÊNCIAS BIBLIOGRÁFICAS}

Araújo FFD, Bragante RJ, Bragante CE. Controle genético, químico e biológico de meloidoginose na cultura da soja. Pesquisa Agropecuária Tropical, v.42, p.220-224, 2012.

Barbosa JC, Maldonado Junior W. AgroEstat: sistema para análises estatísticas de ensaios agronômicos. Jaboticabal, FCAV/UNESP. 396p, 2015.

Conab, 2020. Boletim da safra de grãos. $11^{\circ}$ Levantamento Safra 2019/20- Safra 2019/20. Disponível em: https://www.conab.gov.br/info-agro/safras/graos/boletimda-safra-de-graos. Acesso em 11 dez. 2020.

Costa NL, Santana AC, Coronel DA, Brum AL, Mattos CAC. Aspectos da importância do complexo soja no Brasil e no Rio Grande do Sul: 1997-2017. Redes (St. Cruz Sul, Online), v.25, n.4, p.1840-1863, 2020. http://dx.doi.org/10.17058/redes.v25i4.12735.

Cruz TT, Asmus GL, Garcia RA. Espécies de Crotalaria em sucessão à soja para o manejo de Pratylenchus brachyu$\begin{array}{lll}r u s . \text { Ciência Rural, v.50, n.7, } 2020 . & .\end{array}$ https://doi.org/10.1590/0103-8478cr20190645.
Figueiredo A. Estudo de variáveis ecológicas de Pratylenchus brachyurus em soja e elaboração de uma escala de notas para seleção de genótipos a campo. Ano de obtenção: 2013. 86 f. Tese (Doutorado em Agronomia) - UNESP, Jaboticabal.

Grigolli JFJ, Asmus GL. Manejo de nematoides na cultura da soja. Manejo de nematoides na cultura da soja. Embrapa Agropecuária Oeste-Capítulo em livro científico (ALICE), 2014

Koenning SR, Wrather JA, Kirkpatrick TL, Walker NR, Starr JL, Mueller JD. Plant-parasitic nematodes attacking cotton in the U.S.: old and emerging problems. Plant Disease, v.8, n.2, p.100-113, 2004.

Machado AP, Costa MJN. Biocontrole do fitonematoide Pratylenchus brachyurus in vitro e na soja em casa de vegetação por Bacillus subtilis. Revista Biociências, v.23, n.1, p.83-94, 2018.

Oliveira MKDS, Chaves A., Vieira DADN, da Silva EJ, Walber DDL. Controle biológico de fitonematóides do gênero Pratylenchus através deinoculante natural em cana-de-açúcar. Revista Brasileira de Ciências Agrárias,Recife, v.6, n.2, p.203-207, 2011. DOI:10.5039/agraria.v6i2a835.

Rezende AA. Efficiency of different commercial products with Trichoderma spp. for the control of Sclerotinia stem rot of soybean. 2011. 133 f. Dissertação (Mestrado em Ciências Agrárias) - Universidade Federal de Uberlândia, Uberlândia.

Santos ARB, Almeida FAD, Leite MLT, Fonseca WL, Alcântara Neto FD, Pereira FF, Santos TSD. Biocontrole no manejo de Pratylenchus brachyurus na soja. Revista de Ciências Agrárias, v.42, n.3, p.201-210, 2019. http://dx.doi.org/10.19084/rca.17201.

Silva RA, Nunes NA, Santos TFS, Iwano FK. Efeito da rotação e sucessão de culturas no manejo de nematoides da soja em área arenosa. Nematropica, v.48, n.2, p.198-206, 2018.

Soares R, Dias W. Manejo de nematoides da soja: escolha o milho certo! Embrapa Soja-Fôlder/Folheto/Cartilha (INFOTECA-E), 2020.

Sundararaju P, Jeyabaskaran KJ. Evaluation of different soil types on multiplication of Pratylenchus coffeae and growth of banana seedlings var. Nendran. Nematologia Mediterranea, v.31, n.2, 2003.

Usda - United States Departamente of Agriculture. 2020. World Agricultural Production. Disponível em: https://downloads.usda.library.cornell.edu/usdaesmis/files/5q47rn72z/dz0117445/qv33sf19j/production.pdf> Acesso em: 29 abr. 2020. 